\title{
ON CONTINUED FRACTIONS OF THE FORM
}

$$
1+\stackrel{\infty}{K}_{1}^{\infty}\left(b_{\nu} z / 1\right)
$$

BY H. S. WALL

1. Introduction. The principal object of this paper is to determine the region of convergence of the infinite continued fraction

$$
1+\stackrel{\infty}{K}_{1}^{\infty}\left(b_{\nu} z / 1\right)=1+\frac{b_{1} z}{1}+\frac{b_{2} z}{1}+\ldots, \quad\left(b_{n} \neq 0\right),
$$

when $b_{1}, b_{2}, b_{3}, \cdots$ are real or complex numbers such that for some $k \geqq 1$

$$
\lim _{n=\infty} b_{n k+m}=\sigma_{m}, \quad(m=1,2,3, \cdots, k) .
$$

The results may be stated in terms of the numerators and denominators $u_{n, \lambda}, v_{n, \lambda}$ of the $n$th convergent of the continued fraction $1+K_{\nu=1}^{\infty}\left(\sigma_{\nu+\lambda} z / 1\right),\left(\sigma_{n k+m}=\sigma_{m}\right)$, as follows.

TheOREM 1. Let $\dagger$ us write $G_{k}=v_{k-1,1} v_{k-1,2} \cdots v_{k-1, k}$ and $H_{k}=v_{k}+u_{k-1}-v_{k-1} ;$ and let us set

$$
Z(z)=-(-z){ }^{k} \sigma_{1} \sigma_{2} \cdots \sigma_{k} / H_{k}^{2} .
$$

Let $R$ be an arbitrary bounded closed and connected region of the $z$ plane containing the origin on the interior and which contains (within or upon the boundary) none of the zeros of the polynomials $G_{k}, H_{k}$, nor points z such that $Z(z)$ is a real number $\leqq-1 / 4$. Then (1) converges over $R$ except at certain isolated points $p_{1}, p_{2}, \cdots$, $p_{\mu}$, and uniformly over the region obtained from $R$ by removing the interiors of small circles with centers $p_{1}, p_{2}, \cdots, p_{\mu}$. The limit is a non-rational function of $z$ analytic over $R$ except at $p_{1}, p_{2}, \cdots$, $p_{\mu}$, which are poles.

The function $Z(z)$ determines a transformation of the $z$ plane into the $Z$ plane and $Z=Z(z)$. Except in the case $\sigma_{1} \sigma_{2} \cdots \sigma_{k}$ $=0$, the set of points in the $z$ plane such that $Z$ is real and

$\dagger$ We write $u_{n, 0}=u_{n}$, and $v_{n, 0}=v_{n}$. 
$\leqq-1 / 4$ is a portion of a curve $C_{k}$ to which corresponds under the transformation the real $Z$ segment $(-\infty,-1 / 4)$. The curve $C_{k}$ is a stelloid $\dagger$ or Holzmüller $\ddagger$ hyperbola in the plane of $1 / z$. In particular, $C_{1}$ is a straight line, and $C_{2}$ a circle in the plane of $z$.

In $\$ 2$ we prove Theorem $1 ; \S 3$ contains a discussion of the curves $C_{k}$; and $\$ 4$ contains examples and a discussion of the power series which corresponds to (1).

2. Proof of Theorem 1. If $N_{n}(z) / D_{n}(z)$ is the $n$th convergent of (1), there are $k$ continued fractions

$$
K_{m}=Z_{0}^{m}+\prod_{1}^{\infty}\left(Z_{v}^{m} / 1\right), \quad(m=1,2, \cdots, k),
$$

with convergents $N_{\nu k+m-1} / D_{\nu k+m-1},(\nu=0,1,2,3, \cdots)$, and $\S$

$$
\begin{aligned}
& Z_{0}^{m}=\frac{N_{m-1,0}}{D_{m-1,0}}, \\
& Z_{1}^{m}=\frac{(-1)^{m-1} b_{1} b_{2} \cdots b_{m} z^{m} D_{k-1, m}}{D_{m-1,0} D_{k+m-1,0}}, \\
& Z_{n}^{m}=\frac{-(-z)^{k} b_{1}^{*} b_{2}^{*} \cdots b_{k}^{*} D_{k-1,(n-3) k+m} D_{k-1,(n-1) k+m}}{D_{2 k-1,(n-3) k+m} D_{2 k-1,(n-2) k+m}},
\end{aligned}
$$

for $n \geqq 3$, and where $b_{j}^{*}=b_{(n-2) k+m+j}$ and $N_{n, \lambda} / D_{n, \lambda}$ is the $n$th convergent of

$$
1+\frac{b_{1+\lambda} z}{1}+\frac{b_{2+\lambda} z}{1}+\cdots
$$

By a known theorem, $\|$ if

$$
\lim _{n=\infty} Z_{n}^{m}=Z(z)
$$

uniformly over a region $R^{\prime}$, where $Z(z)$ is a continuous function having nowhere in $R^{\prime}$ a real value $\leqq-1 / 4$, then there exists an index $N$ such that if $n \geqq N$, the continued fraction $K_{\nu=1}^{\infty}\left(Z_{\nu+n}^{m} / 1\right)$ converges uniformly over $R^{\prime}$ to an analytic limit $F_{n}(z)$. Then if

$\dagger$ Fouret, Comptes Rendus, vol. 106 (1888).

$\ddagger$ Holzmüller, Einführung in die Theorie der isogonalen Verwandschaften, 1882, p. 170 and p. 203.

$\S$ Perron, Die Lehre von den Kettenbruchen, 1913, p. 200.

|| Ibid., p. 285. 
$N_{n}^{\prime} / D_{n}^{\prime}$ is the $n$th convergent of $K_{m}$, the latter will converge over $R^{\prime}$ to the limit

$$
\frac{N_{n}^{\prime}+F_{n} N_{n-1}^{\prime}}{D_{n}^{\prime}+F_{n} D_{n-1}^{\prime}},
$$

provided the denominator in (6) is not $\equiv 0$. But if, as we now suppose, $R^{\prime}$ contains the origin on the interior, this is impossible because the denominator $=1$ when $z=0$. Hence $K_{m}$ converges to a function which is analytic except for poles, and clearly converges uniformly in the region obtained from $R^{\prime}$ by removing the interiors of small circles having these poles as centers. Also, $K_{m}$ converges uniformly in the vicinity of the origin. From this it follows $\dagger$ that if $R^{\prime}$ is connected, and if (2) holds for $m=1,2$, $3, \cdots, k$ uniformly over $R^{\prime}$, and $Z(z)$ is independent of $m$, then $K_{1} \equiv K_{2} \equiv \cdots \equiv K_{k} \equiv P(z)$, where $P(z)$ is the power series corresponding to (1), and hence (1) converges after the manner of $K_{m}$ to the same value.

It remains to be shown that under the hypothesis (2), (5) holds over the region $R$ described in the theorem, and that $Z(z)=-(-z)^{k} \sigma_{1} \sigma_{2} \cdots \sigma_{k} / H_{k}^{2}$. We have, if $\delta_{n}=n k+m$,

$$
D_{2 k-1, \delta_{n}}=D_{k-1, \delta_{n+k}} D_{k, \delta_{n}}+\left(N_{k-1, \delta_{n+k}}-D_{k-1, \delta_{n+k}}\right) D_{k-1, \delta_{n}},
$$

and hence, if $D_{k-1, \delta_{n+1}} / D_{k-1, \delta_{n}}=1+\epsilon_{n}=1 /\left(1+\epsilon_{n}^{\prime}\right)$,

where

$$
Z_{n}^{m}=\frac{-(-z)^{k} b_{1}^{*} b_{2}^{*} \cdots b_{k}^{*}}{\Delta_{n-3} \Delta_{n-2}^{\prime}},
$$

$\Delta_{n-3}=D_{k, \delta_{n-3}}+N_{k-1, \delta_{n-2}}-D_{k-1, \delta_{n-2}}+\epsilon_{n-3} D_{k, \delta_{n-3}}$,

$\Delta_{n-2}^{\prime}=D_{k, \delta_{n-2}}+N_{k-1, \delta_{n-1}}-D_{k-1, \delta_{n-1}}+\epsilon_{n-2}^{\prime}\left(N_{k-1, \delta_{n-1}}-D_{k-1, \delta_{n-1}}\right)$.

By (2), $\lim _{n=\infty} \epsilon_{n}=\lim _{n=\infty} \epsilon_{n}^{\prime}=0, \lim _{n-\infty} N_{k, \delta_{n}}=u_{k, m}, \lim _{n=\infty} D_{k, \delta_{n}}$ $=v_{k, m}$ uniformly over $R$. Also

$$
v_{k, m}+u_{k-1, m}-v_{k-1, m} \equiv v_{k}+u_{k-1}-v_{k-1} \equiv H_{k},
$$

for all $m \geqq 1$. It now follows that over the region $R$

$$
\lim _{n=\infty} Z_{n}^{m}=-(-z)^{k} \sigma_{1} \sigma_{2} \cdots \sigma_{k} / H_{m}{ }^{2}, \quad(m=1,2, \cdots, k),
$$

uniformly, as was to be proved.

$\dagger$ Ibid., p. 342. The argument used there applies with slight modification to $K_{m}$ 
In case $\sigma_{1} \sigma_{2} \cdots \sigma_{k}=0$, it is clear that $Z(z) \equiv 0$, and therefore can never be real and $\leqq-1 / 4$. In this case (5) holds uniformly over every bounded region from which the neighborhoods of the zeros of $G_{k}, H_{k}$ have been excluded. It may happen that these neighborhoods need not be excluded. More generally, even if (2) fails to hold, we have the following theorem.

THEOREM 2. If for some integer $k \geqq 1$ the functions $Z_{n}^{m},(m=1$, $2, \cdots, k)$, defined in (3) converge uniformly to 0 for $n=\infty$ over every bounded region, then the continued fraction (1) represents $a$ meromorphic function of $z$ and converges except at the poles of that function.

When $k=1$ this reduces to the condition $\dagger \lim b_{n}=0$ found by E. B. Van Vleck. In $\$ 4$ we shall give examples illustrating this theorem in the cases $k=3,4$.

3. Discussion of the Curves $C_{k}$. Put $p=(-1)^{k} \sigma_{1} \sigma_{2} \cdots \sigma_{k}$, and let $p \neq 0$. By $C_{k}^{\prime}$ we shall understand the set of points in the $z$ plane which is the image of the real $Z \operatorname{segment}(-1 / 4,-\infty)$ under the transformation

$$
Z=-p z^{k} / H_{k}^{2}
$$

Then $C_{k}^{\prime}$ is a portion of a curve $C_{k}$ which is the image of the negative half of the real $Z$ axis under this transformation, and is a cut for the function represented by (1).

If (2) holds, then

$$
\lim _{n=\infty} b_{n q+m}=\sigma_{m}^{\prime}, \quad(m=1,2,3, \cdots, q),
$$

where $q=2 k$ and $\sigma_{m+k}^{\prime}=\sigma_{m}^{\prime}=\sigma_{m}$. If we had started with the hypothesis (8), then instead of the function $Z(z)$ we would have $Z^{\prime}(z)=-p^{2} z^{q} / H_{q}^{2} ;$ and

$$
\begin{aligned}
G_{q} & =G_{k}^{2} H_{k^{q}} \\
H_{q} & =H_{k}^{2}-2 p z^{k} \\
Z^{\prime}(z) & =-\frac{1}{(2+1 / Z(z))^{2}} .
\end{aligned}
$$

In fact, if we let $n=\infty$ in (7), we obtain the relation $v_{q-1, m}$ $=v_{k-1, m} H_{k}$, from which (9) follows at once. From three relations analogous to $(7)$ we obtain the identities

$\dagger$ Ibid., p. 345. 


$$
\begin{gathered}
v_{q}=v_{k}^{2}+v_{k-1}\left(u_{k}-v_{k}\right), \quad v_{q-1}=v_{k} v_{k-1}+v_{k-1}\left(u_{k-1}-v_{k-1}\right), \\
u_{q-1}=u_{k} v_{k-1}+u_{k-1}\left(u_{k-1}-v_{k-1}\right),
\end{gathered}
$$

and consequently $H_{q}=H_{k}^{2}+2\left(u_{k} v_{k-1}-u_{k-1} v_{k}\right)=H_{k}^{2}-2 p z^{k}$, which is (10). Finally, on eliminating $-p z^{k}$ between the relations

$$
Z=-p z^{k} / H_{k}^{2}, \quad Z^{\prime}=-\left[-\frac{p z^{k}}{\left(H_{k}^{2}-2 p z^{k}\right)}\right]^{2},
$$

we obtain (11).

It follows from (11) that $C_{k}^{\prime}$ is the same as $C_{q}^{\prime}$; and from (9) we see that the zeros of $G_{q}$ are those of $G_{k} H_{k}$. When $p \neq 0$, the zeros of $H_{k}$ and of $H_{q}$ lie on the cut; and when $p=0$, the zeros of $H_{q}$ are the same as those of $H_{k}$ by (10). Hence when $k$ is odd in (2) we may turn to (8) instead and obtain precisely the same region of convergence of the continued fraction (1). There is no loss in generality in assuming $k$ even in (2).

In order to identify the curves $C_{k}$ and determine $C_{k}^{\prime}$ it will be convenient to replace $z$ by $1 / z^{\prime}$ and study the corresponding curve $E_{k}$ in the plane of $z^{\prime}=1 / z$, and the portion $E_{k}^{\prime}$ of $E_{k}$ which corresponds to $C_{k}^{\prime}$. If, as we now suppose, $k$ is even, say $=2 q$, then $H_{k}$ is a polynomial of degree $q$ of the form $1+\sum_{1}^{a} A_{\nu} z^{\nu}$. We find that

$$
\frac{(-p)^{1 / 2}}{[Z(z)]^{1 / 2}}=z^{\prime q}+A_{1} z^{\prime q-1}+\cdots+A_{q} .
$$

As $Z(z)$ ranges through real values $\leqq 0,1 /(Z(z))^{1 / 2}$ ranges through pure imaginary values, $z^{\prime}$ over $E_{k}$, and $z$ over $C_{k}$. As $Z(z)$ ranges through real values from $-1 / 4$ to $-\infty, 1 /(Z(z))^{1 / 2}$ ranges through pure imaginary values from $-2 i$ to $+2 i, z^{\prime}$ over $E_{k}^{\prime}$, and $z$ over $C_{l_{k}}^{\prime}$. Set

$$
\begin{gathered}
p^{1 / 2}=\frac{G}{2} e^{i \phi}, \quad A_{\nu}=a_{\nu} e^{i \alpha_{\nu}}, \quad z^{\prime}=r e^{i \theta}, \\
z^{\prime q}+A_{1} z^{\prime q-1}+\cdots+A_{q}=X+i Y,
\end{gathered}
$$

where $\phi$ is any one of the possible arguments of $p^{1 / 2} ; G, a_{\nu}$ are real and positive, and $\alpha_{\nu}, \theta, r, X, Y$ are real. We have 


$$
\begin{aligned}
& X=\sum_{\nu=0}^{q} a_{\nu} r^{q-\nu} \cos \left(\alpha_{\nu}+\overline{q-\nu} \theta\right) \\
& Y=\sum_{\nu=0}^{q} a_{\nu} r^{q-\nu} \sin \left(\alpha_{\nu}+\overline{q-\nu} \theta\right),
\end{aligned}
$$

$\left(a_{0}=1, \alpha_{0}=0\right)$. Let $t$ be real. Then $E_{k}$ is given parametrically by the equations

$$
X=t \cos \phi, \quad Y=t \sin \phi .
$$

On eliminating $t$ we find that $E_{k}$ is the stelloid or Holzmüller hyperbola

$$
X \sin \phi-Y \cos \phi=0,
$$

and that $E_{k}^{\prime}$ is that part of $E_{l}$ for which

$$
X^{2}+Y^{2} \leqq G^{2} \text {. }
$$

If $q=1(k=2), E_{2}$ is a straight line, and (13) is the interior of a circle with center on $E_{2}$. The curve $C_{2}^{\prime}$ is an arc of a circle, and $E_{4}$ is a rectangular hyperbola. In case $q=2, C_{4}$ and $C_{4}^{\prime}$ can be determined by the following special method. First determine $\delta_{1}, \delta_{2}$ by the conditions

$$
\begin{aligned}
& A_{1} \equiv \sigma_{1}+\sigma_{2}+\sigma_{3}+\sigma_{4}=2\left(\delta_{1}+\delta_{2}\right), \\
& A_{2} \equiv \sigma_{1} \sigma_{3}+\sigma_{2} \sigma_{4}=\delta_{1}^{2}+\delta_{2}^{2}
\end{aligned}
$$

so that

$$
\delta_{\nu}=\frac{A_{1}+(-1)^{\nu}\left(8 A_{2}-A_{1}^{2}\right)^{1 / 2}}{4}, \quad(\nu=1,2), \quad 8 \delta_{1} \delta_{2}=A_{1}^{2}-4 A_{2} .
$$

If $\delta_{1} \delta_{2}=0$, then $A_{1}^{2}-4 A_{2}=0$. Hence if either $A_{1}$ or $A_{2}$ is zero, the other is also. If $\delta_{1} \delta_{2} \neq 0$, put

$$
\begin{aligned}
\Delta & =\frac{\sigma_{1} \sigma_{2} \sigma_{3} \sigma_{4}}{\left(\delta_{1} \delta_{2}\right)^{2}}, \\
Z_{1} & =-\frac{\left(\delta_{1} \delta_{2}\right)^{2} z^{4}}{\left[1+2\left(\delta_{1}+\delta_{2}\right) z+\left(\delta_{1}^{2}+\delta_{2}^{2}\right) z^{2}\right]^{2}} .
\end{aligned}
$$

Then $Z=\Delta Z_{1}$. The function $Z_{1}$ is of the form of $Z^{\prime}$ in (11) (for the case $k=4$ ), so that

$$
Z_{1}=-1 /\left(2+1 / Z_{2}\right)^{2}
$$


where $Z_{2}=-\delta_{1} \delta_{2} z^{2} /\left(1+\delta_{1} z+\delta_{2} z\right)^{2}$. When $\delta_{1} \delta_{2}=A_{1}=A_{2}=0$, it is easy to see that $Z=-\sigma_{1} \sigma_{2} \sigma_{3} \sigma_{4} z^{4}$; and when $\delta_{1} \delta_{2}=0, A_{1} A_{2} \neq 0$, $Z=-\sigma_{1} \sigma_{2} \sigma_{3} \sigma_{4} z^{4} / A_{2}^{2}\left(z+2 / A_{1}\right)^{4}$. We find that there are four cases.

CASE 1. $\delta_{1} \delta_{2}=A_{1}=A_{2}=0$. The curve $C_{4}^{\prime}$ consists of four rays running from $z_{n}$ to $\infty$ in the direction from 0 to $z_{n}$, where $z_{n}$, $(n=1,2,3,4)$, are the four fourth roots of $1 /\left(4 \sigma_{1} \sigma_{2} \sigma_{3} \sigma_{4}\right)$. In this case $\sigma_{1} \neq \sigma_{3}$ or else $\sigma_{2} \neq \sigma_{4}$, so that the case $k=2$ is not included.

CASE 2. $\delta_{1} \delta_{2}=0, A_{1} A_{2} \neq 0$. In the plane of $z /\left(z+2 / A_{1}\right)$ the cut consists of four rays as in Case 1 except that the four fourth roots of $A_{2}^{2} /\left(4 \sigma_{1} \sigma_{2} \sigma_{3} \sigma_{4}\right)$ are the initial points of the rays. In the plane of $z$ the cut consists of arcs of two circles. Here $\sigma_{1} \neq \sigma_{3}$ or $\sigma_{2} \neq \sigma_{4}$.

CASE 3. $\delta_{1} \delta_{2} \neq 0, \delta_{1}+\delta_{2}=A_{1} / 2 \equiv 0$. In the plane of $z^{2}$ the cut is an arc of a circle. We may have $\sigma_{1}=\sigma_{3}, \sigma_{2}=\sigma_{4}$ if and only if $\sigma_{1}=-\sigma_{2}$. Thus (2) may hold with $k=2$. The cut consists of two rays running to $\infty$ in this degenerate case.

CASE $4 . \delta_{1} \delta_{2} \neq 0, \delta_{1}+\delta_{2} \neq 0$. We may set $\delta_{1}+\delta_{2}=2 \delta$, and apply (11) (for the case $k=2$ ) to the function $Z_{2} \delta^{2} /\left(\delta_{1} \delta_{2}\right)$. We find that in the plane of $(2+1 /(\delta z))^{2}$ the cut is an arc of a circle. If $\sigma_{1}=\sigma_{3}, \sigma_{2}=\sigma_{4}, \sigma_{1} \neq-\sigma_{2}$, (2) holds with $k=2$, and the cut is an arc of a circle in the plane of $z$. If $\sigma_{1}=\sigma_{2}=\sigma_{3}=\sigma_{4}$, (2) holds with $k=1$ and the cut is the ray running from $-1 /\left(4 \sigma_{1}\right)$ to $\infty$ in the direction from 0 to $-1 /\left(4 \sigma_{1}\right)$.

If the $\sigma_{n}$ are real and positive it is easy to show that $\delta_{1} \delta_{2} \neq 0$, $\delta_{1}+\delta_{2} \neq 0$, so that Case 4 obtains. In this important case one may show that the cut is a portion of the negative half of the real $z$ axis. The polynomial $G_{4}$ is

$$
\left(1+\sigma_{1} z+\sigma_{2} z\right)\left(1+\sigma_{2} z+\sigma_{3} z\right)\left(1+\sigma_{3} z+\sigma_{4} z\right)\left(1+\sigma_{4} z+\sigma_{1} z\right) \text {. }
$$

4. Examples and Applications. The following examples have been selected for the purpose of bringing out interesting points which might otherwise be overlooked.

EXAMPLE 1. Let $b_{3 n-2}=c_{n} \neq 0, \lim c_{n}=0, b_{3 n+2}=-b_{3 n}=a \neq 0$. Here we have, with $k=3$,

$$
\begin{aligned}
& Z_{n}^{1}=-\frac{z^{3} a^{2} c_{n}}{\left(1+c_{n-1} z\right)\left(1+c_{n} z\right)}, \\
& Z_{n}^{2}=-\frac{z^{3} a^{2} c_{n}\left(1+c_{n-1} z\right)\left(1+c_{n+1} z\right)}{\left[1+\left(c_{n}+c_{n-1}\right) z\right]\left[1+\left(c_{n}+c_{n+1}\right) z\right]},
\end{aligned}
$$




$$
Z_{n}^{3}=-\frac{z^{3} a^{2} c_{n}}{\left(1+c_{n} z\right)\left(1+c_{n+1} z\right)}
$$

Since $\lim _{n=\infty} Z_{n}^{m}=0,(m=1,2,3)$, uniformly over every bounded region, the continued fraction represents a meromorphic function of $z$ by Theorem 2 .

EXAMPLE 2. Let $b_{4 n+1}=-b_{4 n+2}=c_{n}>0, \lim c_{n}=0, b_{4 n}=-b_{4 n-1}$ $=a>0$. If $b_{1}=1 / a_{1}, b_{n}=1 /\left(a_{n-1} a_{n}\right),(n \geqq 2)$, then $a_{2 n+1}>0$. Since $\sum a_{2 n+1}$ diverges, it follows from the work of Hamburger $\dagger$ that the continued fraction converges except upon the real axis. To prove that it represents a meromorphic function $\ddagger$ it is but necessary to note $\$$ that when $k=4$

$$
Z_{n}^{1}=-\frac{z^{4} a^{2} c_{n-1} c_{n-2}}{\left(1-a c_{n-2} z^{2}\right)\left(1-a c_{n-1} z^{2}\right)},
$$

so that $\lim Z_{n}{ }^{1}=0$ uniformly over every bounded region, and therefore $K_{1}$ represents a meromorphic function.

Example 3. If $\lim \sup \left|b_{n}\right|<g, \lim _{n=\infty} b_{n} b_{n+1}=0$, we may show that (1) represents a function which is analytic except for poles in the region $\||z| \leqq 1 /(2 g)$. In fact, if $k=2, \lim _{n=\infty} Z_{n}^{m}$ $=0,(m=1,2)$, uniformly over this region inasmuch as the polynomials $D_{3,2 n+m}=1+\left(b_{2 n+m+2}+b_{2 n+m+3}\right) z$ have no zeros in this region if $n$ is sufficiently large; and $\lim _{n=\infty} b_{1}^{*} b_{2}^{*}=0$.

ExAmple 4. According to Theorem 1, the real segment $(1, \infty)$ is to be excluded from the region of convergence of the continued fraction (1) if the $b$ 's have the values $b_{1}=1 / 2, b_{2}=-1 / 2$, $b_{2 n}=-1 /\left(2\left(1+[n-1]^{p} / n^{p}\right)\right), \quad b_{2 n+1}=-1 /\left(2\left(1+[n+1]^{p} / n^{p}\right)\right)$. One may show that this continued fraction converges or diverges at $z=1$ (a point on this segment) according as $p$ is $>1$ or $\leqq 1$. In fact, when $z=1$, the $n$th convergent is

$\dagger$ Mathematische Annalen, vol. 82, pp. 120-187.

‡ See this Bulletin, vol. 39 (1933), pp. 946-952, in which another example is given to show that (1) may represent a meromorphic function when the $b_{n}$ are real and $b_{2 n} b_{2 n+1}>0$, lim sup $\left|b_{n}\right|>0$. In that example convergence was established except at the poles of the function, whereas here the question of convergence at points on the real axis is not considered.

$\S$ The other $Z_{n}^{m},(m=2,3,4)$, are not all so simple in character.

II If the condition $\lim b_{n} b_{n+1}=0$ is dropped, the same holds in the region $|z| \leqq 1 /(4 g)$ (see Perron, loc. cit., p. 343). 


$$
\frac{1}{1^{p}}+\frac{1}{2^{p}}+\cdots+\frac{1}{[n / 2]^{p}}
$$

when $n$ is even and

$$
\frac{1}{1^{p}}+\frac{1}{2^{p}}+\cdots+\frac{1}{[(n-1) / 2]^{p}}+\frac{1}{2[(n-1) / 2]^{p}}
$$

when $n$ is odd.

As is well known, (1) has a unique corresponding power series $P(z)=\sum c_{\nu} z^{\nu},\left(c_{0}=1\right)$, from which (1) may be obtained by a repeated division process. If a given power series $P(z)$ has a corresponding continued fraction of the form (1), it is said to be semi-normal. $\dagger$ When convergent, the continued fraction furnishes a method for summing the power series. Let $P^{\prime}(z)$ $=\sum_{\nu=0}^{\infty} c_{1+\nu} Z^{\nu}$ be semi-normal with corresponding continued fraction $c_{1}+K\left(b_{\nu}^{\prime} z / 1\right)$. Then if (1) converges to $f(z)$ when (1) corresponds to $P(z)$, it is important to know conditions under which $c_{1}+K\left(b_{\nu}^{\prime} z / 1\right)$ converges to the value $(f(z)-1) / z$.

It is well known that if (1) converges to $f(z)$, then when $c_{1}+K\left(b_{\nu}^{\prime} z / 1\right)$ converges it must have the value $(f(z)-1) / z$. This follows from the fact that the even convergents of $1+c_{1} z+z K\left(b_{\nu}^{\prime} z / 1\right)$ are the same as the odd convergents of (1). $\ddagger$

The numbers $b_{\nu}$ and $b_{\nu}^{\prime}$ are related as follows. $\$$ Set $a_{1}=1 / b_{1}$, $a_{1}^{\prime}=1 / b_{1}^{\prime}, a_{n}=1 /\left(b_{n} a_{n-1}\right), a_{n}^{\prime}=1 /\left(b_{n}^{\prime} a_{n-1}^{\prime}\right),(n>1)$. Then if $h_{n}=a_{1}+a_{3}+\cdots+a_{2 n+1}$,

$$
a_{2 n}^{\prime}=a_{2 n+1} /\left(h_{n} h_{n-1}\right), \quad a_{2 n+1}^{\prime}=a_{2 n+2} h_{n}^{2} .
$$

If (2) holds with $k$ even ( $\operatorname{say}=2 q$ ), then it is not difficult to show that when the $b_{n}$ are real and

$$
\lim _{n=\infty}\left(b_{n k+m} / b_{n k+m+1}\right)=r_{m}>0, \quad(m=1,2,3, \cdots, k),
$$

we must also have

$$
\lim _{n=\infty}\left(b_{n k+m}^{\prime} / b_{n k+m+1}^{\prime}\right)=r_{m}^{\prime}>0, \quad(m=1,2,3, \cdots, k),
$$

$\dagger$ Perron, loc. cit., p. 304.

$\ddagger$ Perron, loc. cit., p. 447.

$\S$ Transactions of this Society, vol. 31 (1929), pp. 102-103. 
and

$$
\lim _{n=\infty} b_{n k+m}^{\prime}=\sigma_{m}^{\prime}, \quad(m=1,2,3, \cdots, k) .
$$

Hence if Theorem 1 is applicable to the continued fraction corresponding to $P(z)$ it is also applicable to the continued fraction corresponding to $P^{\prime}(z)$ provided $P(z)$ has real coefficients and (15) holds. From (16), (17) it then follows at once that Theorem 1 can be in turn applied to the continued fraction corresponding to $P^{\prime \prime}(z)=\sum_{\nu=0}^{\infty} c_{2+\nu} z^{\nu}$, provided the latter is semi-normal, etc. It is easy to conclude from the fact that two successive continued fractions obtained in this way have an infinite number of convergents in common that the function $Z(z)$ of Theorem 1 is the same for all these continued fractions. We shall summarize the result in the following theorem.

TheOREM 3. If $P(z)=1+c_{1} z+c_{2} z^{2}+\cdots$ is a semi-normal power series with real coefficients and corresponding continued fraction (1) such that for $k=2 q$ equations (2) and (15) hold, and if $L=c_{1}+K\left(b_{\nu}^{\prime} z / 1\right)$ is the corresponding continued fraction for $P^{\prime}(z)=c_{1}+c_{2} z+c_{3} z^{2}+\cdots$ (supposed semi-normal), then (1) and $1+z L$ converge to one and the same function $f(z)$ over the region $R$ described in Theorem 1. If $P^{(n)}(z)=\sum_{\nu=0}^{\infty} c_{n+\nu} z^{\nu}$ is seminormal with corresponding continued fraction $c_{n}+K\left(b_{\nu}^{(n)} z / 1\right)$, $(n=1,2,3, \cdots, r)$, then all the continued fractions $1+c_{1} z$ $+\cdots+c_{n} z^{n}+z^{n} K\left(b_{\nu}^{(n)} z / 1\right), \quad(n=1,2,3, \cdots, r)$, converge over $R$ to $f(z)$.

The continued fractions are precisely the continued fractions of "type 1" of a Padé table, $\uparrow$ whose convergents are "stairlike" files of approximants beginning upon the horizontal side of the table. One can show that when $E(z)$, the reciprocal of $P(z)$, and the series $E^{(n)}(z)$ obtained by removing from $E(z)$ the first $n$ terms and the factor $z^{n},(n=1,2,3, \cdots)$, are seminormal, then under the hypothesis of Theorem 3 the continued fractions corresponding to stairlike files of approximants beginning on the vertical side of the table also converge over $R$ to $f(z)$.

NORTHWESTERN UNIVERSITY

$\dagger$ Perron, loc. cit., pp. 447-448. 